\title{
Ultrafast Laser-Pump Electron-Probe Microscopy for Imaging Semiconductor Carrier Dynamics
}

\author{
Mathijs W.H. Garming ${ }^{1}$, I.G. (Gerward) C. Weppelman ${ }^{1}$, Pieter Kruit ${ }^{1}$, and Jacob P. Hoogenboom ${ }^{*}$ \\ ${ }^{1 .}$ Imaging Physics, Delft University of Technology, Delft, The Netherlands \\ ${ }^{*}$ Corresponding author: j.p.hoogenboom@tudelft.nl
}

Laser-pump electron-probe microscopy is a unique technique to unravel semiconductor charge carrier dynamics at the ns time scale. As opposed to all-optical pump-probe techniques, ultrafast electron probing could bring sub-micrometer resolution and high surface sensitivity, properties desirable for the inspection of nanoscale solid state devices.

Pioneering work of the Zewail group has shown it possible to study semiconductor carrier dynamics by photoexciting semiconductor materials and probing the carrier induced change of secondary electron (SE) yield in a scanning electron microscope (SEM) [1]. A pump probe scheme where carriers are created with a fs laser pulse and subsequently detected with an electron pulse arriving with adjustable delay, can therefore be used to image carrier dynamics in space and time, with resolution down to tens of microns demonstrated thus far [2].

Here, we further develop the laser pump electron probe technique for studying charge carrier dynamics. Our FEI Quanta SEM, illustrated in Figure 1, is based setup is equipped with two laser pump paths, one to bring 25 fs laser pulses in a few tens of microns excitation spot on non-transparent substrates, the other makes use of an inverted optical microscope $[3,4]$ to focus laser pulses into a sub-micron spot. Electron pulses are generated by beam blanking, allowing for 90 ps pulses at $50 \mathrm{~nm}$ spatial resolution [5]. We use lock-in detection to separate processes occurring on the ns time scale from those that go beyond the laser repetition time.

Proof of principle measurements on semiconductor materials indeed show a time dependent change in SE yield when the delay between laser pump and electron probe pulses is varied on the ns time scale. As shown in Figure 2, slower processes can also be detected with the use of lock-in amplification. We found the SE detection scheme is highly sensitive to surface morphology as different crystal orientations of the sample result in different observed dynamics. Additionally, sub-micron spatial resolution of this technique is demonstrated; by the high-NA laser excitation, and by mapping carrier dynamics around metal nanostructures on a GaAs substrate.

\section{References:}

[1] Cho et al., PNAS 111 (2014), p. 2094.

[2] Liao et al., Nano Lett. 17 (2017), p. 3675.

[3] Moerland et al., Nano Lett. 18 (2018), p. 6107.

[4] Garming et al., Nanoscale 9 (2017), p. 12727.

[5] Moerland et al., Optics Express 24 (2016), p. 24760. 


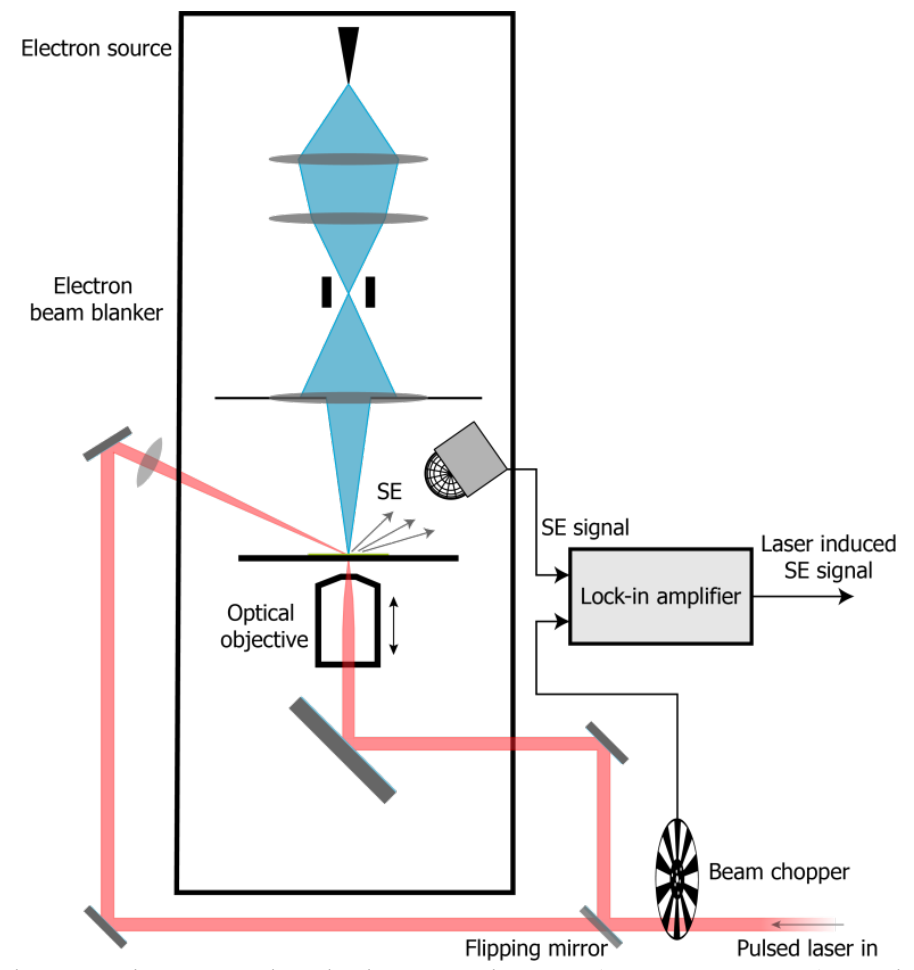

Figure 1. The SEM based setup has a pulsed electron beam (90 ps, $50 \mathrm{~nm})$ and two possible paths for laser excitation of the sample. Laser pump electron probe measurements are conducted by scanning the pulsed electron beam around the fs laser spot to acquire spatiotemporal information. Lock-in detection is for enhanced signal and the detection of slow processes.

\section{Laser induced SE signal GaAs (100)}
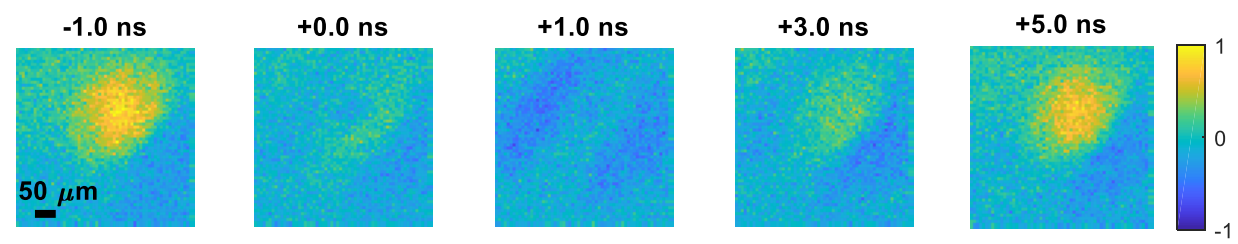

Figure 2. The laser induced SE yield for different laser pump electron probe delays shows a decrease in $\mathrm{SE}$ yield right after laser excitation at $\mathrm{t}=0 \mathrm{~ns}$ with $\mathrm{ns}$ scale recovery; carrier presence causes a reduction of SE yield for this material. The signal recovers to a positive value; a bright spot develops that is still visible before the next laser pump pulse. This indicates the presence of a process slower than the $10 \mathrm{~ns}$ pump probe repetition time, hypothesized to be carrier trapping. 